\title{
Identifying Sound Pedagogical Practices Based on Findings from Neuroscience
}

\author{
Chandana Watagodakumbura ${ }^{1, *}$ \\ ${ }^{1}$ Faculty of Information Technology, Monash University, Melbourne, Australia \\ *Correspondence: Faculty of Information Technology, Monash University, Melbourne, \\ Australia. E-mail: chandana.watagodakumbura@monash.edu
}

Received: March 25, 2015 Accepted: April 22, 2015 Published: May 4, 2015

doi:10.5296/ije.v7i2.7318 URL: http://dx.doi.org/10.5296/ije.v7i2.7318

\begin{abstract}
We have identified goals of education by viewing them from the point of neuroscience; through education, we have to produce individuals who are better problem solvers and decision makers. To achieve this goal, learners will have to transform what they have learned explicitly into implicit memories and vice versa. Further, through education, we enhance learner consciousness and wisdom. A number of pedagogical practices that are useful in achieving the above goals are presented. When new contents are presented in a teaching-learning environment, high-level concepts need to be highlighted; the concepts are likely to penetrate through multiple domain areas thus helping learners to form better neural networks of knowledge. In order to reach out to multiple brain regions, we need to get the frontal lobe involved essentially and hence the pace of presentation has to be controlled appropriately; as the frontal lobe connects to many brain regions, the processing occurs relatively slowly. The important task of motivating learners can be done by presenting learners with neuroscience-based facts about learning; even difficult content can be mastered by simply paying attention elaborately; human brains have the feature of plasticity and through learning, neural networks can grow throughout the lifespan. Taking into consideration the phenomenon of binocular rivalry - human brains can concentrate only on one thing at a time fully- we should encourage learners to engage in the discussion in a teaching-learning session fully. When setting assessment, we should focus on open-ended, novel conceptual questions so that learners use their frontal lobes connecting many other regions as well.
\end{abstract}

Keywords: neuroscience, learning, pedagogical practices, implicit and explicit learning, goals of education, visual-spatial learners, consciousness, creativity 


\section{Introduction}

This paper presents a number of useful pedagogical practices we can use as educators by looking at education from the viewpoint of neuroscience. It is organised into three main sections - sections 2, 3 and 4. In section 2, we present the neuroscience-based concepts that are related to learning in general. Section 3 highlights goals of education when we look at them from the point of view of neuroscience. How the goals of education presented in section 3 can be achieved in teaching-learning environments and assessments is elaborated in section 4 of the paper. A brief concluding summary follows in section 5 .

\section{Introduction to Neuroscience-based Concepts that Relate to Pedagogical Practices}

\subsection{Basic structure of the human brain (Baars and Gage, 2010)}

Mainly there are 3 layers of the brain, from bottom to top: the reptilian brain, mammalian brain and neocortex. The reptilian brain is the oldest layer of the brain; it is composed of the brain stem, the structures that dominate in the brains of snakes and lizards. This part of the brain controls survival activities such as breathing, hart rate and balance. The mammalian brain is layered over the reptilian brain and consists of a system of brain parts called the limbic system. The constituents of the limbic system include amygdala, hippocampus and hypothalamus. The limbic system plays a major role in human emotions. The $3^{\text {rd }}$ layer of the brain is the neocortex or primate brain which is the most recent addition to our brain. It consists of wrinkled covering of cerebral hemispheres - left and right. The neocortex plays a major role in cognitive, linguistic, motor, sensory and social abilities. It gives considerable flexibility in creativity in adapting to changing environments. The neocortex is densely interconnected with the limbic system and controls the expression of emotions.

The neocortex has 4 major lobes, namely, the frontal lobe, parietal lobe, temporal lobe and occipital lobe. One significant feature of the brain structure is brain localisation. That is, the brain is composed of a large number of functionally specialised regions. There are about 100 Brodmann areas, so to speak, now recognised in the neocortex. The 4 lobes of the neocortex and their processes are intricately intertwined with each other. Further, the neocortex, which is vital for cognitive functions, interacts constantly with major so called satellite organs such as the thalamus, basal ganglia, cerebellum, hippocampus and limbic regions.

Interestingly, the right and left cerebral hemispheres of the neocortex have differentiated functions. The left hemisphere is more specialised in performing verbal and linguistic, mathematical, sequential and analytical functions while the right hemisphere is more inclined to perform non-verbal, spatial, geometrical, musical and synthesis functions. The 2 cerebral hemispheres are connected together by the largest fibre bundle in the brain- corpus callosum.

It is of special interest to know that frontal lobes are referred to as the organ of civilisation. The role the frontal lobes play in cognition is uniquely human and without their development civilisation could never have arisen. They are crucial for all higher-order purposeful 
behaviour such as identifying the objective, projecting the goal, forging plans to reach it, organising the means by which such plans can be carried out and monitoring and judging the consequences. Further, frontal parts of the brain become active when resolving conflicting conditions. A correlation has also been identified between frontal activation and longer reaction time and sense of subjective effort. In summary, the frontal lobe, or more specifically the pre-frontal cortex (PFC), is used for human activities such as language, thought and executive control of higher-order processes and connect directly with every distinct functional unit of the brain. This connectivity allows PFC to coordinate and integrate the functions of other brain structures.

\subsection{Types of memory (Baars and Gage, 2010)}

Human memory can be defined as a lasting representation that is reflected in thought, experience, and/or behaviour. It can be divided in to 2 main types: explicit and implicit. Explicit memory refers to the memory with conscious awareness and the individual can declare its existence and comment on its content either verbally or non-verbally. Consequently, such memories are known as declarative memories. On the other hand, implicit memory is not accompanied by conscious awareness that one has a memory; the existence of implicit memory is inferred only from the effects it has on behaviour. Further, implicit memories may be retained without an intention to remember and accessed commonly by priming tasks.

Explicit or declarative memory can be further divided into 2 types: episodic (autobiographical) memory and semantic memory. Episodic memory refers to memories that have a specific source in time, space and life circumstances. In contrast, semantic memories involve facts (or high-level concepts and generalisations) about us, the world, and other knowledge that we share within a community and are independent of the spatial and temporal context in which they were acquired. Further, episodic memories are remembered consciously and susceptible to forgetting while semantic memories give a feeling of knowing rather than a fully conscious recollection and less susceptible to forgetting. Initially memories are episodic and context dependent and over time they are transformed into semantic memories.

\subsection{Types of learning from the viewpoint of neuroscience (Baars and Gage, 2010)}

Learning can be defined as the acquisition of lasting representations that involve a wide range of brain areas and activities. Very often, the unstated goal of learning is to turn explicit problem solving into the implicit kind. Similar to the way we discussed about explicit and implicit memories, learning can also be explicit or implicit. Explicit, episodic or declarative learning involves conscious learning while implicit (unconscious) learning results as a side effect of conscious input. That is, even for implicit learning, conscious events guide the learning process. But there is no exclusively conscious learning as both, conscious and unconscious, processes always go together. In a complete learning cycle, 3 phases can be identified: learning, retention and retrieval. Retention is generally viewed as unconscious, although it is shaped by conscious experiences. Explicit learning generally occurs when we pay attention to new information so that it becomes conscious. The brain begins learning as soon as it is placed in any novel environment. Simple novelty is enough to trigger attention 
and learning, including significant evoked potentials that sweep through the entire cortex. As soon as we experience or understand the new information with enough clarity, our brains are able to store it. Sometime it may require repeated attention new or difficult information in order for us to get a sense of clarity. Any new material may seem vague or hard to understand at first; however, when we spend time thinking about it or paying attention to it, a clearer sense of meaning tends to appear.

Brain takes more time to solve novel problems and voluntary actions become automatic with practice. As they do so, we tend to lose executive control over them. Cortical activity reduces when predictable voluntary action is practiced to the point of automaticity. Once even very complex processes are learned, they seem to require less cortical activity.

Most of our learning is identified to be incidental (Eide \& Eide, 2004), meaning that it occurs as a result of paying attention and becoming conscious of. That is, we do not deliberately memorise things all the time; memorising is only one way to make learning happen.

With our current practices, academic learning is mostly explicit with teachers pointing out the things to be learned and students doing their best to memorise them. However, most ordinary human learning is implicit. For example, social habits and language are mostly leant implicitly. Looking from another perspective, most knowledge is tacit knowledge and most learning takes place implicitly before it can be stated explicitly. Further, academic exams usually test associative recall (What is the capital of Australia?) rather than recognition tests. Associative recall tests give much lower estimates for accurate memories than recognition tests. That is, in associative recall, we expect more exact and specific answers than in recognition tests. Interestingly, these exact and specific answers are the ones that are likely to be forgotten soon.

In the event of some emotional stimuli, there is evidence that unconscious learning takes place. That is, this gives much stronger evidence for implicit learning, in which some inferential process takes conscious input and encodes unconscious results. In other words, emotional learning results in implicit emotional memory that retains classically conditioned emotional relationships that cannot be voluntarily recollected or reported. Psychological evidence shows that moderate levels of emotional arousal at the time of an event lead to better retention of explicit memories (Sylwester, 1998). That is, explicit memories are better consolidated by the reception of emotional stimuli by the amygdala.

Hebbian learning, named after neuropsychologist Donald Hebb, is summarised to "neurons that fire together wire together". It indicates that the more frequent certain synaptic connections are made the more likely they are to form lasting neural networks. In other words, synaptic connections that are rarely used will eventually die out (Diamond, 1996, 2001). This is a very simple idea on how we can explain the way learning takes place. In fact, it is observed that forming new synaptic connections or synaptogenesis takes place throughout one's lifespan, enabling a lifelong learning process. In other words, cortical plasticity lasts throughout the lifespan of a human being. However, in a changing, dynamic world, unlearning also has an important role to play. That is, we will have to let misconceptions or inaccurate knowledge to die out from our neural system. Further, human brains are identified 
to be selectionist rather than being instructionist, meaning that synaptic connections can grow by selecting new connections, not merely being restricted by an existing limited set of instructions as that happens in computers. In other words, human brains can be creative by learning new knowledge in the form of new synaptic connections that did not exist before.

\subsection{Consciousness from the viewpoint of neuroscience (Baars and Gage, 2010)}

Scientists have confirmed that consciousness can be defined and studied scientifically, contrary to the beliefs held previously. Consciousness results from neuronal interaction between thalamocortical systems; the neocortex is the main organ involved in it. The evolution of consciousness is understood to be the highest expression of the developed brain that parallels the evolution of the prefrontal cortex (PFC). Synonyms used for consciousness are awareness, explicit cognition and focal attention. Consciousness can be identified mainly in two levels - primary and higher-order consciousness. The former is concerned with perceptual world while the latter is related to abstractions and thought. Further consciousness involves with a range of contents: sensory perception, visual imagery, emotional feelings, inner speech, abstract concepts and action related ideas indicating the involvement of a number of brain regions, through an integrative view. Of special interest is the theory developed by Giulio Tononi in the name of Integrated Theory of Consciousness. It provides a new way to study consciousness using a rigorous scientific approach. Integrated theory of consciousness is a framework that is built on the notion that consciousness is a consequence of systems that have both a large amount of differentiated information that is also highly integrated. To summarise the idea, a computer may have a large quantity of memory (say 16 GB), but since these memory pieces are not integrated, computers do not have consciousness. Scientists have also been able to quantify the level of consciousness with a measure called neural complexity (C). High values of $\mathrm{C}$ characterise conscious events and reflects the extent to which the dynamics of a neural system are both integrated and differentiated. Consciousness in humans can also be understood with the cognitive architecture known as Global Workspace Theory (GWT). GWT proposes that momentarily dominant information is widely distributed in the brain. That is, the nervous system can be viewed as a massive distributed set of special-purpose networks. Consequently, coordination, control, and novel problem solving could take place by way of central information exchange. Conscious involvement of brain resources is particularly useful when novel information needs to be combined and integrated.

\subsection{Attention from the viewpoint of neuroscience (Baars and Gage, 2010)}

The word attention seems to imply the ability to direct cognitive resources to some event. It has a kind of pointing or directive sense. Selective attention implies a choice among a number of possible events. Consciousness seems to be the experience of an event after it has been selected by paying attention. We can decide voluntarily what to become conscious of or the selection can also be automatic if stimuli are intense, dynamic or biologically or personally important. In the real world, voluntary and automatic attentions are generally mixed. In summary, attention is defined as the ability to select information for cognitive purposes. This selection may be shaped by emotion (Zull, 2002), motivation and salience and is at least 
partly under executive control. The term binocular rivalry is used to describe the process of selective attention in neuroscience. When 2 items are looked at the same time using one eye on each item using a pair of binoculars, at an instance of time we can only see one item properly or consciously. In other words, we cannot concentrate well on 2 things at the same time even though we see them both simultaneously. That is, when a person is given a task that requires in-depth, meaningful analysis of the material, memory under divided attention is much worse than memory under full attention. Deeper processing requires time to complete and divided attention limits the time allotted to encoding. In learning, what we generally do is just pay attention to new material, even if it seems hard to understand. The biggest challenge is to pay continued attention to new and difficult information and to be patient enough to allow our brains to do wonder, ask questions, and ultimately comprehend any new material.

\subsection{Automaticity from the viewpoint of neuroscience (Baars and Gage, 2010)}

In general, the more predictable a sensorimotor skill becomes, the less of it will become conscious. The fading of conscious access to habitual skills is commonly called 'automaticity' and it goes along with a loss of precise voluntary control over habitual details. Repetitive events tend to fade from consciousness unless they have special significance. That is, voluntary actions we are conscious with become automatic with practice. As they do so, we tend to lose executive control over them. In other words, effortful tasks show a wider spread of brain activity; the brain takes more time to solve novel problems and switching from one task to another seems to require additional mental resources beyond those involved in routine and automatic actions. The level of activity in cortex (at least) drops with practice and automaticity. It seems to indicate the recruitment of neuronal resources that are needed to work together to perform a task that is new or unpredictable.

\subsection{Types of problem solving/decision making from the viewpoint of neuroscience (Baars and} Gage, 2010)

Individuals need to become better decision makers and problem solvers through education. In schools or colleges, we are usually given a problem or question and we must find or write the correct answer. Usually only one correct answer exists to these questions or problems. For example, balancing a check book and remembering the capital city of a country are similar tasks. By finding the correct solution, we engage in veridical decision-making. However, apart from high school exams, college tests and factual and computational trivia, most decisions we make in our everyday lives do not have intrinsically correct solutions. That is, the decisions we make are not always objective, rather they are, in most cases, subjective. What career path to make, what location to visit in the vacation are some such example decisions we make in ambiguous situations. By making a decision or choice, we engage in adaptive decision-making. Further, our best neural system performance is not for the exact symbol sequences that conventional computers handle so well. Rather, our brains are exceptionally good at dealing with complex, ill-defined, and novel challenges, the kinds that people have to deal with in real world. That is, humans are exceptionally flexible in adapting to new conditions. The choices we make are not inherent in the situations at hand. There are complex interplay between the properties of the situations and our own individual properties, 
aspirations, doubts, and histories. The prefrontal cortex (PFC) is central to such decision-making or evaluation. Finding solutions for deterministic situations often is accomplished algorithmically or following a number of steps routinely. These tasks are increasingly delegated to various devices such as calculators, computers and the like. However, making judgments, in the absence of inherently correct solutions, remain, at least for now, a uniquely human territory. Thus, through education an individual must develop the capacity to have the flexibility to adopt different perspectives on the same situation at different times. The organism must be able to disambiguate the same situation in multiple different ways and to have the capacity to switch between them at will. Frontal lobes of the brain hemispheres play an important role in dealing with these ambiguous situations.

It appears that goals of and approaches to science education have shifted recently. Science usually works from a third person perspective. This means that researchers adopt an objective point of view, seeing all evidence as a physical object. Even human beings are seen as objects. Recently, scientists interested in consciousness have begun arguing for an additional way of conducting and approaching science that appreciates and accepts data gathered from first person perspective, i.e. phenomenological data from introspection or self-report. In second person perspective, the other person is viewed as a subject rather than an object, as someone who has mental states.

\section{Reflecting on Goals of Education Taking into Consideration Findings from Neuroscience (Baars and Gage, 2010)}

Broadly, the main goal of education is to develop human beings into better problem solvers and decision makers who achieve a deeper perception of reality through enhanced creativity and wisdom. Wisdom is understood here as an advanced from of creativity (Claxton, 2008) that penetrates into knowledge of multiple domain areas. From the basis of neuroscience, enhancing wisdom means enhancing consciousness. As presented in the Integrated Theory of Consciousness(Baars and Gage, 2010), we can understand consciousness as the integration of a large number of differentiated information or brain regions that possibly span multiple domain areas of knowledge. In other words, we aim to develop interconnected, denser neuronal networks in individuals as they evolve through learning. Further, by enhancing consciousness, we say we achieve human development (Dabrowski, 1970, 1972, 1977) into self-actualising (Maslow, 1968, 1993) human beings. This process of self-actualisation or human development can be a lifelong process; it indicates the additional goal of education in which individuals need to learn self-reliance in accommodating lifelong learning. As we can see, we have a broader goal of education that goes beyond merely training individuals to specific career paths. In the theory of multiple intelligences (Gardner, 2006), we have identified a number of intelligences a human being can possess. In our main goal of education, we want individuals to develop as many areas of intelligences across multiple brain areas. In short, we want individuals to develop holistically, as opposed to in a restricted, one-sided manner.

In a teaching-learning environment, learners engage in explicit learning (Baars and Gage, 
2010); teacher gives instructions on what to learn and learners attempt to grasp what is instructed. When novel material is presented long enough, possibly from diverse perspectives, the contents become clearer to learners and we say the brain has achieved magical learning. Now, if clarity is achieved to a high degree, what is learned explicitly becomes implicit, meaning that our brain can implicitly or unconsciously apply the concepts learned in a similar but different situation. In relation to a teaching-learning environment, the goal of education is to transform explicit learning to implicit learning. This process of transformation should continue endlessly; that is, once a number of concepts are transformed, from explicit to implicit memories, we do not stop there; rather, we look for more concepts for similar transformation. The plasticity feature of our brain plays an important role here; neuronal networks in human brains can grow endlessly, explaining the concept of creativity associated with human beings, compared to non-creative machines or computers (Beale, R. and T. Jackson, 1990). In other words, human brains are selectionist, compared to computers, which are instructionist.

Most of our learning is identified to be implicit (Baars and Gage, 2010); that is we learn from day to day life situations we encounter by simply paying attention. In these situations, we do not learn explicitly in a selected domain per se, but retain traces of implicit memories in diverse domains. More specifically, we have evidence that gifted individuals learn implicitly or incidentally more naturally (Eide \& Eide, 2004). That is, learners bring wealth of implicit memories or experiences to a classroom environment, albeit to different degrees depending on personal traits. Consequently, another goal of education is to transform these implicit memories to more explicit or declarative memories. Then, learners will be able to use and express this tacit knowledge more elaborately when the circumstances arise.

We live in a neurodiverse (Armstrong, 2011) society; every individual is neurologically and psychologically different from others. Some learners are identified to demonstrate visual-spatial abilities while others demonstrate auditory-sequential abilities more predominantly. The former category of learners is likely to be identified as gifted individuals (Silverman, 1998, 2002; Webb, 2005, 2008) who usually demonstrate overexcitable characteristics (Dabrowski, 1970, 1972, 1977) such as emotional, intellectual and imaginational. Taking into consideration this broad concept of neurodiversity, a goal of education is to provide a uniform, systematic and fair form of education to every individual, despite their inherent differences. Achieving this elusive goal of education can be a real challenge in a contemporary social environment.

In the following sections, we look at some important pedagogical practices through which we can reach out achieving the above mentioned goals of education.

\section{Application of Findings from Neuroscience in Our Pedagogical Practices/ Classroom Environments}

\subsection{Presenting new content to learners in a teaching-learning environment}

When presenting new contents to learners in a teaching-learning environment, we need to 
emphasise on the high-level concepts we present instead of on more specific details. High-level concepts are retained in memory longer as semantic memory, which is independent of the time and space contexts (Baars and Gage, 2010). On the other hand, specific details are stored as episodic memory which is more susceptible to forgetting; further, these specific details usually have references to time and space. Since high-level concepts are usually more generalised knowledge, they tend to penetrate through multiple domain areas as well. Consequently, by emphasising on high-level concepts, we not only create more lasting memories, but also enable the interconnection of knowledge in multiple domain areas. To reiterate, to enhance wisdom in learners, we have to let them create knowledge across multiple domains.

Further, when we introduce new high-level concepts, we have to help learners to link them to their existing knowledge bases (Zull, 2002). Existing knowledge bases are most likely to be concepts based on day to day life phenomena across multiple domains. By doing this, we allow them to create new neural networks and link them existing neural networks. In this way, we support them to build more lasting integrated knowledge bases, instead of isolated pieces that are susceptible for forgetting. In addition, when new concepts are presented, we can guide and get learners to link the new concepts themselves to their existing personalised body of knowledge. That is, learners attempt to identify relationships between existing and new knowledge while we encourage them to do so themselves. The result is that learners engage in the process of creating knowledge as highlighted in the theory of constructivist theory (Biggs, 2003) and do so relatively autonomous manner.

In order for learners to identify the mentioned relationships, we can guide them by asking a series of questions in guiding towards reaching the target; learners will get to the target by answering or attempting to answer these questions. Further, we can make use of related analogies or anecdotes to direct learners towards the target. The key point here is that we make them identify the relationships between existing and new knowledge themselves rather than we relate to them in order for them to commit to memory, which is unlikely to give a lasting impact. Even if we present a well-established theory to our learners, we should mimic the thinking process that would have gone through in the mind of the creator of this theory; the creator may have self-inquired himself/herself in numerous ways before confirming the knowledge. We should lead our learners in a similar path of self-inquiry mimicking a process of construction of knowledge.

\subsection{Controlling the pace of presentation appropriately for learners to construct knowledge}

We need to present new contents to learners in a teaching-learning environment in a manner that they can link them to their existing knowledge bases (Zull, 2002). These existing knowledge bases or neural networks may have been spread across multiple regions of the brain, depending on individual neurological and psychological differences (Watagodakumbura, 2013). To reach out to these multiple regions, we need to essentially send messages to the frontal lobes, which are highly connected to all the other regions (Baars and Gage, 2010). However, when knowledge is processed in the frontal lobes and connects with the knowledge in the other regions, this whole task takes relatively longer time, or the 
process is slower. This could be due to the parallel processing that takes place within multiple brain regions. Also, we have to remember that frontal lobes are considered as the organ of civilisation; that is any processing involving frontal lobes or resulting higher-order processing has the impact of a deeper and better learning experience. Consequently, when presenting new contents to learners, we have to present them at an appropriate pace taking into account the relative slowness in processing that involves the frontal lobes. Looking from another point of view, frontal lobes are usually involved with the process of abstract conceptualisation (Kolb, 1983); as a result, when new contents are presented by means of introducing new concepts, we need to allow learners adequate time to understand or conceptualise them. This contrasts from presenting contents to learners rapidly so that they have to commit them to short term memory in order for them to reproduce in the exact form later on, not necessarily getting the frontal lobes involved appropriately.

\subsection{Motivating learners to engage in deeper learning by giving facts from neuroscience}

In a teaching-learning environment, for effective learning to take place, learners have to engage well in the learning process. Ideally, they have to be intrinsically motivated in learning; that is, they see some long lasting value in learning, not only for the purpose of getting through forthcoming examinations or their future careers, but in general in everyday lives. In order for us to motivate our learners to engage deeply in the learning process, we can provide some useful facts about learning related to neuroscience. Our brains use more brain resources in learning new material (Baars and Gage, 2010) and learning new material is relatively more engaging. However, even difficult contents can be mastered by simply paying attention for a longer period of time; then the brains do the wonder of learning - making lasting connections with the existing knowledge base. In addition, our brains have the property of plasticity that helps us to create new neural connections or networks altogether. That is we can be creative and develop wisdom if we try to understand new concepts deeply by linking them to other existing concepts or frameworks. This task of learning is a lifelong process that would not end at the completion of examinations or formal studies. As we move on with our lives, we develop more integrated, useful neural networks that cross the boundaries of domain areas, thus enhancing consciousness and wisdom. In this way, we can become better problem solvers and decision makers by seeing the reality better, as an evolutionary process over the years of our life spans. By giving positive facts about learning and contents presented, we can create positive emotions such as pleasure in the minds of learners. At the same time, we can get rid of any negative emotions such as fear (Zull, 2002) from the minds of learners with appropriate instructions and behaviour; for example if learners get the feeling that the contents are not difficult to master, if a systematic approach is persisted, they may not be fearful of them; if the teacher presents a non-authoritative figure, as that of a facilitator, in front of the class, learners will be more comfortable with no negative emotions. These positive emotions lacking the negative ones help learners more specifically to be motivated in the learning process.

\subsection{Getting learner attention fully on the teaching-learning process or discussion}

Neuroscientists have identified a concept referred to as binocular rivalry (Baars and Gage, 
2010) that explains that human brain can concentrate or pay attention only on one thing completely at a time. We can apply this principle to our teaching-learning environment by asking learners to only listen by paying full attention and engage in the discussion. Learners can be provided with summaries of the discussion and if possible a voice/video recording of the same for their reference later on. Still, if they want to write down some notes in their own words, we can provide note taking time separately for this purpose. We are trying to avoid learners undergoing the situation of divided attention that would have a negative impact on deeper learning. By doing this, we encourage learners to be fully vigilant by paying attention to the discussion during which time they construct meaning or perform abstract conceptualisation (Kolb, 1983) by linking new knowledge to the existing knowledge base.

\subsection{Useful considerations when setting up assessment}

When setting up assessment questions, we need to consider getting involved learners frontal lobes that connects multiple brain regions, in providing answers. By doing this, we are able to test how well learners were able engage in deep learning (Biggs, 2003; Entwistle, 1998) by constricting individualised meaning (Yero, 2002). To achieve this objective, we can ask relatively novel and open-ended conceptual questions (Watagodakumbura, 2013). We deviate from asking questions that require regurgitation of factual and exact recalled information. In other words, we encourage learners to engage in adaptive decision making instead of veridical decision making even when answering examination questions. We ask questions from learners in order to discourage them from providing premeditated or habitual answers. That is, if they have answered a similar question before, they may provide a habitual answer; the brain plays an automaticity function (Baars and Gage, 2010) here bypassing the engagement of cortical resources including the frontal lobe appropriately in providing answers.

We would like to emphasise that the discussion in the above paragraph can be applied well even to science education, not only for social science education. Science usually works from the third person perspective, adopting an objective point of view (Baars and Gage, 2010). However, recently there is a shift in seeing science from a first person perspective, especially by the scientists interested in studying consciousness. In this approach, phenomenological data is seen from introspection or self-report. Consequently, what we have discussed in the preceding paragraph can be applied to science education environments equally well encouraging learners to develop creativity and wisdom.

\section{Conclusion}

At the broadest level, the goal of education is identified as producing individuals who are better problem solvers and decision makers, not only in their careers, but also in everyday life activities (Watagodakumbura, 2013); thus individuals get to see the reality better or as is. In other words, individuals develop a higher level of consciousness or wisdom. In order to achieve this goal, we have to stimulate as many brain regions of a learner as possible; in this regard, we have to get the frontal lobes, the organ of civilisation, essentially involved during 
the process of teaching-learning and subsequent assessment. Since the frontal lobes connect many other brain regions, we encourage learners to integrate knowledge from multiple domain areas, thus enhancing their wisdom. To practically achieve this higher-order learning that enhances consciousness and wisdom in a teaching-learning environment, we have to highlight high-level concepts that are stored as semantic memory; semantic memory lasts longer compared to episodic memory that relates to more specific details. Further, in assessment, we have to focus on open-ended and novel and high-level concepts-based questions in order to put learners on a higher-order learning path as well as to maintain the validity of our assessment, in a lasting manner. Importantly, through the emerging evidences from neuroscience, we, the educators, can now get guided in our pedagogical practices to provide a better learning experience to our learners; here, we are guided by scientific facts and biologically what is better for our learners to evolve in the process of human development into creative human beings with wisdom. Further, through these evidence-based practices, we promote inclusive practices in a neurodiverse (Armstrong, 2011) society; the disadvantaged situations faced by gifted and creative individuals or visual-spatial learners (Silverman, 1998, 2002) in a traditional environment will be reduced significantly; numerous psychological issues faced by gifted individuals (Webb, 2005, 2008) demonstrating overexcitable characteristics (Dabrowski, 1970, 1972, 1977) will be minimised.

\section{References}

Armstrong, T. (2011). The Power of Neurodiversity: Unleashing the Advantages of Your Differently Wired Brain. Da Capo Lifelong Books.

Baars, B. J., \& Gage, N. M. (2010). Cognition, Brain, and Consciousness - Introduction to Cognitive

Beale, R., \& T. Jackson. (1990). Neural Computing - An Introduction. Institute of Physics Publishing. http://dx.doi.org/10.1887/0852742622

Biggs, J. (2003). Teaching for Quality Learning at University ( $2^{\text {nd }}$ ed.). Buckingham, Society for Research into Higher Education and Open University Press.

Claxton, G. (2008). Wisdom: Advanced Creativity? In A. Craft, H. Gardner \& G. Claxton (Eds.) Creativity, Wisdom and Trusteeship: Exploring the Role of Education. Thousand Oaks, CA: Corwin Press.

Dabrowski, K. (1972). Psychoneuroses Is Not An Illness. London: Gryf Publications.

Dabrowski, K. (1977). Theory of Levels of Emotional Development (vol 1) - Multilevelness and Positive Disintegration. New York: Dabor Science Publications.

Dabrowski, K. (with Kawczak A., \& Piechowski M. M.). (1970). Mental Growth through Positive Disintegration. London: Gryf Publications.

Diamond, M. C. (1996). The Brain... Use it or Lose It. New Horizons for Learning: School of Education, Johns Hopkins University. Retrieved from 
http://education.jhu.edu/newhorizons/Neurosciences/articles/

Diamond, M. C. (2001). Response of the Brain to Enrichment. New Horizons for Learning: School of Education, Johns Hopkins University. http://dx.doi.org/10.1016/B0-08-043076-7/03626-3

Eide, B. \& Eide, F. (2004). 'Brains on Fire: The Multimodality of Gifted Thinkers', New Horizons for Learning: School of Education, Johns Hopkins University. Retrieved from http://education.jhu.edu/PD/newhorizons/Neurosciences/articles/

Entwistle, N. J. (1998). Approaches to Learning and Forms of Understanding. In Teaching and Learning in Higher Education, ed. B. Dart and G. Boulton-Lewis, 72-101. Melbourne, Australia: Australian Council for Educational Research.

Gardner, H. (2006). Multiple Intelligences: New Horizons. New York: Basic Books.

Kolb, D. (1983). Experiential Learning: Experience as the Source of Learning and Development, Upper Saddle River, NJ: Prentice Hall.

Maslow, A. (1968). Toward a Psychology of Being. New York: Van Nostrand Reinhold.

Maslow, A. (1993). Farther Reaches of Human Nature. New York, N.Y., U.S.A.: Arkana. Neuroscience (2nd Ed.). Elsevier, MA: USA.

Silverman, L. K. (2002). Upside-Down Brilliance: The Visual-Spatial Learner. Denver: DeLeon Publishing.

Silverman, L.K. (1998). Personality and Learning Styles of Gifted Children. In Excellence In Educating Gifted \& Talented Learners ( ${ }^{\text {rd }}$ ed), ed. Van Tassel - Baska, Denver, Colorado, USA: Love Publishing Company.

Watagodakumbura, C. (2013b). Education from a Deeper and Multidisciplinary Perspective - To a Sustainable Development of a Neurodiverse Society - A Futuristic View, Xlibris.

Webb, J. T. (2008). Dabrowski's Theory and Existential Depression in Gifted Children and Adults. Paper presented at the Eighth International Congress of the Institute for Positive Disintegration in Human Development, August 7-9, Alberta, Canada.

Webb, J.T. (with Amend E. R., Webb N.E., Goerss J., Beljan P, and Olenchak F.R.) (2005). Misdiagnosis and Dual Diagnoses of Gifted Children and Adults: ADHD, Bipolar, Ocd, Asperger's, Depression, and Other Disorders. Great Potential Press.

Yero, J. (2002). How Teacher Thinking Shapes Education. New Horizons for Learning: School of Education, Johns Hopkins University. Retrieved from http://education.jhu.edu/PD/newhorizons/Neurosciences/articles/

Zull, J. E. (2002). The Art of Changing the Brain: Enriching the Practice of Teaching by Exploring the Biology of Learning. Stylus Publishing. 


\section{Copyright Disclaimer}

2015, Vol. 7, No. 2

Copyright for this article is retained by the author(s), with first publication rights granted to the journal.

This is an open-access article distributed under the terms and conditions of the Creative Commons Attribution license (http://creativecommons.org/licenses/by/3.0/). 